\title{
Resting heart rate associates with one-year risk of major adverse cardiovascular events in patients with acute coronary syndrome after percutaneous coronary intervention
}

\author{
Shao-Li Wang ${ }^{1}$, Cheng-Long Wang ${ }^{2}$, Pei-Li Wang ${ }^{2}$, Hao $\mathrm{Xu}^{2}$, Jian-Peng Du${ }^{2}$, Da-Wu Zhang ${ }^{2}$, \\ Zhu-Ye Gao ${ }^{2}$, Lei Zhang ${ }^{2}$, Chang-Geng Fu ${ }^{2}$, Ke-Ji Chen ${ }^{2}$ and Da-Zhuo Shi ${ }^{2}$ \\ ${ }^{1}$ Guang'anmen Hospital, China Academy of Chinese Medical Sciences, Beijing 100053, China; ${ }^{2}$ Institute of Cardiovascular Disease, \\ Xiyuan Hospital, China Academy of Chinese Medical Sciences, Beijing 100091, China \\ Corresponding authors: Da-Zhuo Shi and Ke-Ji Chen. Email: dazhuo_shi@163.com
}

\begin{abstract}
The study was to access the association between resting heart rate (RHR) and one-year risk of major adverse cardiovascular events (MACE) in acute coronary syndrome (ACS) patients after percutaneous coronary intervention (PCI). Patients with ACS after $\mathrm{PCl}(n=808)$ were prospectively followed-up for MACE. RHR was obtained from electrocardiogram. MACE was defined as a composite of cardiac death, nonfatal recurrent myocardial infarction, ischemic-driven revascularization, and ischemic stroke. The association between RHR and one-year risk of MACE was assessed using Cox proportional hazards regression model. Compared with patients with $\mathrm{RHR}>76 \mathrm{bpm}$, the adjusted hazard ratio (AHR) was 0.51 (95\% confidence intervals [Cl]: $0.23-1.14 ; P=0.100)$ for patients with $\mathrm{RHR}<61 \mathrm{bpm}$, and $0.44(95 \% \mathrm{Cl}$ : $0.23-0.85 ; P=0.014)$ for those with RHR $61-76 \mathrm{bpm}$. For patients with $\mathrm{RHR} \geq 61 \mathrm{bpm}$, an increase of $10 \mathrm{bpm}$ in RHR was associated with an increase by $38.0 \%$ in the risk of MACE (AHR: 1.38; 95\% $\mathrm{Cl}$ : 1.04-1.83; $P=0.026)$. ACS patients after $\mathrm{PCl}$ with RHR $>76 \mathrm{bpm}$ were at higher risk of MACE during one-year follow-up compared with patients with RHR 61-76 bpm. An elevated RHR $\geq 61$ bpm was associated with increased risk of one-year MACE in ACS patients.
\end{abstract}

Keywords: Resting heart rate, acute coronary syndrome, percutaneous coronary intervention, major adverse cardiovascular events

Experimental Biology and Medicine 2016; 241: 478-484. DOI: 10.1177/1535370215617563

\section{Introduction}

An association between resting heart rate (RHR) and cardiovascular events has been found. It was reported that RHR was correlated with the risk of cardiovascular disease both in the general population, ${ }^{1}$ and in patients with a wide spectrum of cardiovascular disease, that consisted of stable coronary artery disease (CAD), ${ }^{2,3}$ heart failure, ${ }^{4}$ as well as acute coronary syndrome (ACS). ${ }^{5}$ However, there seemed to be very few prospective studies that focused on the population of full spectrum of ACS undergoing primary percutaneous coronary intervention (PCI), and assessed the prognostic implication of RHR change at the time of $72 \mathrm{~h}$ after onset of ACS during hospitalization. ${ }^{6}$ Whether an elevated RHR was linked with increased risk of major adverse cardiovascular events (MACE) in ACS patients after PCI during oneyear follow-up remained uncertain. The aim of this study was to access prospectively the association between RHR and one-year risk of MACE in ACS patients after PCI.

\section{Methods}

Study design and patient population

The prospective cohort study that recruited 808 patients was conducted at 13 hospitals in mainland China. The population comprised patients aged 18-75 years who hospitalized for $\mathrm{ACS},{ }^{7,8}$ either acute myocardial infarction (AMI) (with or without ST segment elevation) or unstable angina (UA), and underwent successful PCI (defined as the target vessel with TIMI grade 3 flow). Patients would be excluded if they were concomitantly afflicted with severe complications including cardiogenic shock or heart failure, hepatic dysfunction, kidney failure, hematopoietic dysfunction, psychiatric disorders, cancers, pregnancy or breastfeeding, or concurrently enrolled in other clinical trials. The patients were recruited from April 2008 to October 2009 and each of them was supposed to be followed up for one year. The last patient's follow-up was completed by October 2010. The study protocol was approved by the ethics review board of Xiyuan 
Hospital, China Academy of Chinese Medical Sciences (CACMS) in accordance with the principles described in the Declaration of Helsinki, ${ }^{9}$ and all patients provided written informed consent before enrollment.

\section{Intervention}

All patients received conventional treatment decided by physicians who were not involved in the study. Angiographic follow-up was performed during the follow-up, but was not required in this study.

\section{RHR}

The RHR of the body (commonly called RHR) is the number of contractions of the heart that occur in a single minute while the body is at complete rest. RHR was calculated in the supine position in the morning before getting out of bed with 12-lead electrocardiography on $72 \mathrm{~h}$ after onset of ACS during hospitalization. To improve the accuracy of the measurement, movement during the procedure, exercise and smoking prior to the procedure were prohibited for patients. Generally, fasting is not required before the test. All medications (prescribed and over-the-counter) and herbal supplements were notified by doctors. The average HR per minute was determined by the experienced cardiologists. If disagreement occurred, a panel of cardiologists made the final decision.

\section{Endpoints}

The endpoint in this study was MACE, defined as a composite of cardiac death, nonfatal recurrent MI, ischemiadriven revascularization, and ischemic stoke during one year after successful PCI. All deaths were considered cardiac unless an unequivocal non-cardiac cause was identified. Recurrent MI was diagnosed based on reappearance of symptoms, and/or new electrocardiographic changes in association with a re-elevation of creatine kinase-MB (CK$\mathrm{MB})$ to the level greater than three times the upper limit of the reference level. Ischemia-driven revascularization was defined as repeat revascularization with either PCI or coronary artery bypass graft (CABG) because of recurrent myocardial ischemic events. Ischemic stroke was defined as the development of disabling neurologic symptoms and objective findings lasting at least $24 \mathrm{~h}$.

\section{Data collection and follow-up}

The baseline data was collected and recorded in the case report form (CRF) by the trained investigators, which included demographic characteristics such as age and gender, occupation, education, smoking and drinking habits, medical history, medications, physical examination results, and related test results.

Follow-up visits were scheduled at 30 days and 3, 6, 9, and 12 months after enrollment. The endpoint outcomes were collected and recorded in the CRF by the investigators at each visit (either a direct visit or telephone interview when appropriate). For remote patients via the phone, patients' medical records on endpoint outcomes were requested from the local hospital or outpatient facility and death certificates were obtained from the family. All clinical outcomes were adjudicated by an independent outcome committee with review of original documentations. The monitoring committee reviewed the records at regular intervals to examine events.

\section{Statistical analysis}

We divided RHR into tertiles and the study population was presented in tertiles of RHR. Considering the relatively small sample size of the study, the patients were finally divided into three categories according to their RHR[patients with $\mathrm{RHR}<$ first tertile (T1), with RHR between T1 to second tertile (T2), and with RHR >T2]: (a) $\mathrm{RHR}<61$ beats per minute (bpm) ( $<\mathrm{T} 1, n=181)$, (b) RHR 61-76 bpm (T1-T2, $n=432)$, (c) RHR > 76bpm (>T3, $n=195)$. Baseline demographic and clinical characteristics were compared across RHR subgroups. Continuous variables following normal distribution were presented as mean (with standard deviation) and compared with oneway analysis of variance. Variables that did not follow normal distribution were presented as median (with lower and upper quartiles) and compared with the Mann-Whitney test. Categorical variables were expressed as frequency and percentage and compared with Pearson's Chi-square test or Fisher extract test when appropriate. Univariate associations of RHR with MACE were examined via Kaplan-Meier curves, using the logrank test for comparisons between subgroups. The multivariate Cox proportional hazards regression model was performed to assess the predictive value of RHR for MACE at one-year follow-up. The dependent variable was the rate of one-year MACE, and the independent variable was RHR that was evaluated as a categorical variable. Considering the relatively small number of MACE in the present cohort study, the selection of covariables must be limited. Initially, all the variables, that differed significantly across RHR subgroups or univariatedly associated with MACE with a $P<0.1$ in the univariate analysis, were involved in multivariate Cox proportional hazards regression model, and those with no significant association with MACE in backward stepwise regression were excluded. Finally only RHR and variables that were demonstrated to be independent risk factors for MACE were entered into the multivariable analysis. The results were presented as hazard ratios (HR) with 95\% confidence intervals (CI). The adjusted HR for each subgroup of RHR was calculated in reference to the subgroup with $\mathrm{RHR}<61 \mathrm{bpm}$, where the HR was considered as 1 . In a post hoc analysis, the multivariate analysis with $\mathrm{RHR} \geq 61 \mathrm{bpm}$ and $<61 \mathrm{bpm}$, as a continuous variable, was also performed in the multivariable Cox proportional hazards regression model with the same variables, respectively.

Two-tailed tests of significance were performed. For all comparisons, a $P<0.05$ was considered statistically significant. When appropriate, CI was calculated with a 95\% confidence level. Statistical analyses were performed using the SPSS $^{\mathrm{TM}}$ software package (version 17.0). 


\section{Results}

\section{Patient characteristics}

A total of 808 consecutive patients were included in this study with a median age of 60 years (31-76). $74.6 \%$ of the patients were male. Of the patients, $72.2 \%$ were admitted with Non-ST segment elevation ACS (NSTE-ACS) and $27.8 \%$ with STEMI. The median value of RHR was $69 \mathrm{bpm}$ (38-129 bpm).

During one-year follow-up, three patients died of cardiac events, two patients died of cancer (both due to lung cancer), and 38 patients were classified as dropout. Among the dropouts, five declined to participate in the follow-up, two had non-cardiac adverse events (cancer), 31 were unreachable for data collection due to changing address or phone number, and none of patients were excluded because of the concurrent enrollment in other clinical trials. Finally, a total of 765 patients survived and completed the one-year follow-up. Baseline characteristics of the total population are presented in Table 1. Except percentage of subtype ACS diagnosed at admission and left ventricular ejection fraction (LVEF), there were no significant differences in terms of gender, age, blood pressure (BP) level, the numbers of diseased vessels, target vessels, medical history, smoking history, family history of CAD, body mass index (BMI), left ventricular end-diastolic dimension (LVEED), and medications between different RHR subgroups.

\section{One-year MACE}

During one-year follow-up, $3(0.4 \%)$ patients died from cardiac events, $9(1.1 \%)$ patients experienced recurrent MI, 30 (3.7\%) patients received ischemic-driven revascularization,

Table 1 Baseline characteristics of participants according to RHR at study entry

\begin{tabular}{|c|c|c|c|c|c|}
\hline Covariates & \multicolumn{4}{|l|}{ RHR tertiles (bpm) } & $P$ value \\
\hline Male & $603(74.6)$ & $136(75.1)$ & $321(74.3)$ & $146(74.9)$ & 0.973 \\
\hline Age, years & $60(31,76)$ & $61.4(9.3)$ & $59.4(9.7)$ & $59.6(10.0)$ & 0.068 \\
\hline \multicolumn{6}{|l|}{ Subtype ACS } \\
\hline NSTE-ACS & $583(72.2)$ & $140(77.3)$ & $325(75.2)$ & $118(60.5)$ & $<0.001$ \\
\hline Systolic & $130(85,180)$ & $130(85,180)$ & $130(90,180)$ & $130(85,180)$ & 0.845 \\
\hline Diastolic & $80(60,110)$ & $80(60,110)$ & $80(60,110)$ & $80(60,110)$ & 0.709 \\
\hline \multicolumn{6}{|c|}{ Number of diseased vessels } \\
\hline One & $214(26.5)$ & $50(27.6)$ & $120(27.8)$ & $44(22.6)$ & \multirow[t]{2}{*}{0.247} \\
\hline Two & $246(30.4)$ & $53(29.3)$ & $139(32.2)$ & $54(27.7)$ & \\
\hline RCA & $465(57.5)$ & $105(58.0)$ & $240(55.6)$ & $120(61.5)$ & 0.370 \\
\hline LM & $66(8.2)$ & $15(8.3)$ & $36(8.3)$ & $15(7.7)$ & 0.962 \\
\hline \multicolumn{6}{|l|}{ Medical history } \\
\hline Hypertension & $509(63.0)$ & $113(62.4)$ & $274(63.4)$ & $122(62.6)$ & 0.963 \\
\hline Diabetes & $234(29.0)$ & $44(24.3)$ & $127(29.4)$ & $63(32.3)$ & 0.223 \\
\hline Hyperlipidemia & 322 (39.9) & $80(44.2)$ & $171(39.6)$ & $71(36.4)$ & 0.301 \\
\hline Stroke & $57(7.1)$ & $12(6.6)$ & $31(7.2)$ & $14(7.2)$ & 0.968 \\
\hline Smoking history & $459(56.8)$ & $103(56.9)$ & $242(56.0)$ & $114(58.5)$ & 0.849 \\
\hline CAD family history & $198(24.5)$ & $45(24.9)$ & $94(22.2)$ & 57 (29.2) & 0.167 \\
\hline $\mathrm{BMI}, \mathrm{kg} / \mathrm{m}^{2}$ & $25.4(5.0,39.9)$ & $25.2(2.8)$ & $25.5(2.8)$ & $25.6(3.4)$ & 0.511 \\
\hline
\end{tabular}

Age, blood pressure, body mass index, left ventricular end-diastolic dimension, left ventricular ejection fraction are expressed as Median (min, max) or mean $\pm \mathrm{SD}$. The other values are expressed as numbers (\%).

ACEls, angiotensin-converting enzyme inhibitors; ARBs, angiotensin receptor blockers; BMI, body mass index; BP, blood pressure; BBRs, beta-blockers; CCBs, calcium channel blockers; LAD, left anterior descending artery; LCX, left circumflex artery; LM, left main coronary artery; LVEDD, left ventricular end-diastolic dimension; LVEF, left ventricular ejection fraction; RCA, right coronary artery. 
and $9(1.1 \%)$ patients experienced ischemic stroke. Overall, MACE occurred in 45 (5.6\%) patients. Among subgroups, MACE occurred in $5 \%$ of patients with RHR $<61 \mathrm{bpm}, 4.4 \%$ of patients with RHR between $61 \mathrm{bpm}$ and $76 \mathrm{bpm}$, and $8.7 \%$ of patients with RHR $>76 \mathrm{bpm}$ (Table 2 ).

\section{Univariate analysis}

In univariate analysis, the Kaplan-Meier curve for MACE occurrence among RHR subgroups showed that patients in the subgroup with RHR $>76 \mathrm{bpm}$ had a significantly higher incidence of MACE as compared to patients with RHR between $61 \mathrm{bpm}$ and $76 \mathrm{bpm}(P=0.027)$. However, there was no significant difference in the risk of MACE between the subgroups with RHR $<61 \mathrm{bpm}$ and RHR between $61 \mathrm{bpm}$ and $76 \mathrm{bpm}(P=0.776)$, nor between the subgroups with RHR $<61 \mathrm{bpm}$ and $\mathrm{RHR}>76 \mathrm{bpm} \quad(P=0.134)$ (Figure 1).

\section{Multivariate analysis}

The univariate risk factors of MACE with a $P<0.1$ were target vessel of left main coronary artery (LM), LVEDD, and medications of angiotensin receptor blockers (ARBs)

Table 2 Multivariate analysis using Cox proportional hazards regression testing the relation between RHR and one-year MACE

\begin{tabular}{|c|c|c|c|c|c|}
\hline \multirow[b]{2}{*}{ RHR (bpm) } & \multirow[b]{2}{*}{$N$} & \multirow[b]{2}{*}{ Number of MACE $n$ (\%) } & \multicolumn{3}{|c|}{ Multivariable model } \\
\hline & & & AHR & $95 \% \mathrm{Cl}$ & $P$ value \\
\hline$<61$ & 181 & $9(5.0)$ & 0.51 & $(0.23,1.14)$ & 0.100 \\
\hline $61-76$ & 432 & $19(4.4)$ & 0.44 & $(0.23,0.84)$ & 0.014 \\
\hline$>76$ & 195 & $17(8.7)$ & \multicolumn{2}{|c|}{ Reference } & 0.009 \\
\hline
\end{tabular}

The HR was adjusted by target vessel of LM, LVEDD, medications of ARBs or CCBs, subtype ACS diagnosed at admission (i.e., UA, NSTEMI and STEMI) and LVEF.

AHR, adjusted hazard ratio; RHR, resting heart rate; MACE, major adverse cardiovascular events.

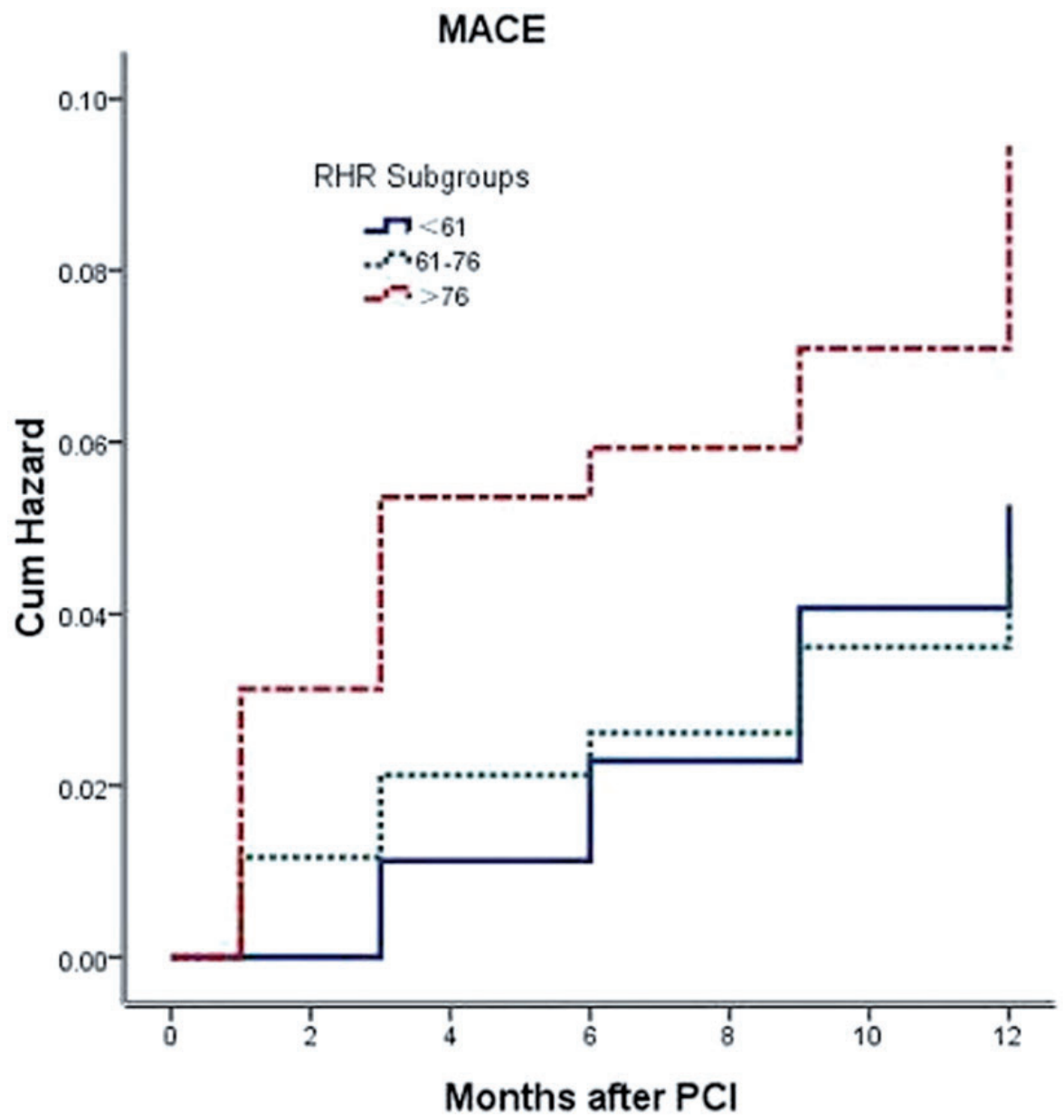

Figure 1 Cumulative incidence of MACE in RHR subgroups. Kaplan-Meier estimates of cumulative incidence of MACE during follow-up for RHR subgroups (<61, $61-76$, and $>76$ bpm). (A color version of this figure is available in the online journal.) 
or calcium channel blockers (CCBs). Initially the multivariate Cox model was adjusted by target vessel of LM, LVEDD, medications of ARBs or CCBs, subtype ACS diagnosed at admission and LVEF using the method of backward stepwise, and variables including LVEDD, target vessel of LM and medications of ARBs or CCBs were entered into the model as covariates to assess the association of RHR and MACE after one-year follow-up. The results showed that the AHR was 0.83 (95\% CI: $0.38-1.85 ; P=0.654$ ) for patients with RHR between 61 and $76 \mathrm{bpm}$, and 1.92 (95\% CI: 0.864.32; $P=0.113$ ) for patients with RHR $>76 \mathrm{bpm}$, as compared with patients with RHR $<61 \mathrm{bpm}$, after adjusting for the covariates. When compared with patients with RHR $>76 \mathrm{bpm}$, the AHR was $0.44(95 \% \mathrm{CI}, 0.23$ to 0.84 ; $P=0.014)$ for patients with RHR between 61 and $76 \mathrm{bpm}$ (Table 2).

In the post hoc analysis, an increasing trend of MACE with increased RHR was apparent in patients with RHR $\geq 61 \mathrm{bpm}$. For the patients with RHR $\geq 61 \mathrm{bpm}$, an increased $10 \mathrm{bpm}$ in RHR was associated with an overall $38.0 \%$ increase in the risk of MACE (AHR: 1.38; 95\% CI: 1.041.83; $P=0.026$ ). However, a non-significant reverse correlation was found between RHR and MACE among patients with RHR <61 bpm (AHR: 0.06; 95\% CI: 0.003-1.34; $P=0.076$ ) (Table 3).

\section{Discussion}

This study demonstrated that RHR calculated on $72 \mathrm{~h}$ after onset of ACS and successful PCI is associated with MACE during one-year follow-up. The patients with RHR $>76 \mathrm{bpm}$ is at higher risk of suffering from MACE compared with those with RHR between 61 and $76 \mathrm{bpm}$. For patients with RHR $\geq 61 \mathrm{bpm}$, elevated RHR was associated with increased risk of one-year MACE.

RHR has long been regarded as an independent predictor of cardiovascular risk among the healthy and various patient populations. ${ }^{10-12}$ Very few studies, however, have addressed the association between RHR and MACE in ACS patients after $\mathrm{PCI}^{13,14}$ especially in Asian populations. The OPUS-TIMI 16 trial demonstrated that higher HR was strongly predictive of increased mortality in patients with ACS. ${ }^{13}$ A more recent study by Ana Teresa Timóteo et al. ${ }^{14}$ demonstrated that one-year all-cause mortality increased progressively with RHR $\geq 80 \mathrm{bpm}$ in patients with ACS (with and without ST-segment elevation). Our findings in the present study was consistent with the observations conducted by the two previous studies, ${ }^{13,14}$ and also showed that elevated RHR $\geq 61 \mathrm{bpm}$ was associated with increased risk of MACE in ACS patients after PCI. The results of our study also paralleled to other findings reported previously in the population of AMI patients. ${ }^{15-17}$ In the SPRINT study, ${ }^{16}$ patients with $\mathrm{HR}>90 \mathrm{bpm}$ who received thrombolytic therapy had a $15.1 \%$ in-hospital mortality compared with $9.5 \%$ for those with HR $70-89 \mathrm{bpm}$. In the GISSI-2 study, ${ }^{17}$ patients with $\mathrm{HR}<60 \mathrm{bpm}$ who treated with fibrinolytic therapy had an in-hospital mortality of $7.1 \%$, whereas patients with $\mathrm{HR}>100 \mathrm{bpm}$ had a $23.4 \%$ mortality. Likewise, for patients with STEMI receiving PCI, Louisa Antoni et al. ${ }^{15}$ demonstrated patients with $\mathrm{HR} \geq 70 \mathrm{bpm}$ had a two-fold increased risk of cardiovascular mortality at one- and four-year follow-up compared with patients with HR $<70 \mathrm{bpm}$. Guido Parodi et al. ${ }^{18}$ showed that an elevated $\mathrm{HR} \geq 80 \mathrm{bpm}$ was associated with increased risk of mortality compared with HR $60-79 \mathrm{bpm}$. Lorenzo Fácila et al. ${ }^{19}$ observed that a RHR $\geq 70 \mathrm{bpm}$ in patients who survive from AMI is an indicator of high risk of cardiovascular events during one-year follow-up.

To our knowledge, this is the first prospective study to evaluate the association of RHR at the time of $72 \mathrm{~h}$ after onset of ACS and PCI during hospitalization and risk of MACE in mainland China. And we have documented the consistent results with previous studies in different patient populations. However, this study did differ from most of the previous studies in two aspects. Firstly, the population in the present study, comprising ACS patients with NSTEACS (72.2\%) and STEMI (27.8\%), were all treated with successful PCI. As such, the characteristics of our study population were different from those of the ACS patients in the OPUS-TIMI-16 trial ${ }^{13}$ and the study performed by Ana Teresa Timóteo et al. ${ }^{14}$ Secondly, in order to classify groups into lower and higher risk of adverse outcome, the RHR calculation in our study was divided into three categories $(<61,61-76,>76 \mathrm{bpm})$ based on tertile stratification of RHR $(<\mathrm{T} 1, \mathrm{~T} 1-\mathrm{T} 2,>\mathrm{T} 3)$, which in accordance with a recent study. ${ }^{20}$

Previous studies have shown that a decrease in RHR using drugs, such as BBRs or ivabradin, had an improvement on the prognosis of patients. Of these studies, Kjekshus et al. ${ }^{21}$ observed BBRs played an important part in reducing total mortality and sudden cardiac death after MI. A recent meta-regression analysis, ${ }^{22}$ including randomized clinical trials of BBRs and CCBs in post-acute MI patients, also demonstrated a relationship between RHR reduction and decreases in risk of death and non-fatal MI. This analysis strongly suggested the beneficial effects of these agents were proportionally related to the reduction of RHR. In our study, an elevated RHR of $\geq 61 \mathrm{bpm}$ was

Table 3 Post hoc analysis for Cox proportional hazards regression testing the relation between RHR in 10 bpm and one-year MACE

\begin{tabular}{llllll}
\hline & & & \multicolumn{3}{l}{ Multivariable model } \\
\cline { 3 - 5 } RHR (bpm) & $\boldsymbol{N}$ & Number of MACE & $\boldsymbol{n}(\%)$ & AHR & $\mathbf{9 5 \%} \mathbf{C l}$ \\
\hline RHR $\geq 61$ & 627 & $36(5.7)$ & 1.380 & $(1.040,1.830)$ \\
RHR $<61$ & 181 & $9(5.0)$ & 0.062 & $(0.003,1.340)$ & 0.026 \\
\hline
\end{tabular}

The HR was adjusted by target vessel of LM, LVEDD, medications of ARBs or CCBs, subtype ACS diagnosed at admission (i.e. UA, NSTEMI, and STEMI) and LVEF. AHR, adjusted hazard ratio; MACE, major adverse cardiovascular events; RHR, resting heart rate. 
also correlated with the risk of MACE in the multivariable analysis after adjusting for use of BBRs and other potentially factors, indicating that patients with increased RHR were at higher risk of one-year cardiovascular events regardless of BBRs use.

Epidemiological data indicated that patients with lower RHR had a better prognosis compared with those with higher $\mathrm{HR}^{23}$ In the CRUSADE study, the relationship between hospital morbidity/mortality and HR followed a J-shaped curve. Very low and high HR was associated with worse outcomes, where, HR of $60-69 \mathrm{bpm}$ had the lowest all-cause mortality. ${ }^{24}$ Similarly, our study demonstrated patients with RHR $<61 \mathrm{bpm}$ had a possible increased risk of MACE (5.0\%) in comparison with those RHR ranging from 61 to $76 \mathrm{bpm}(4.4 \%)$, although the difference was not significantly. As there were only 808 patients enrolled in the present study, the number of cases is likely to be insufficient to detect differences between RHR subgroups in terms of adverse outcomes. Other studies have also found nonlinear associations between RHR and subsequent ischemic heart disease mortality, ${ }^{8,20,25}$ suggesting that both high and low RHR was closely associated with adverse consequences.

The pathophysiological mechanism of RHR related mortality is still elusive. One possibility is that RHR strongly and directly associated with arterial rigidity and impacts directly on the status of the arterial wall. ${ }^{26}$ The other scenario is that RHR decrease improves diastolic perfusion time, while increasing RHR by atrial pacing in patients with CAD leads to the coronary vasoconstriction, possibly further impairing oxygen supply in the body. ${ }^{27}$

There are some limitations of this study. First, the total of 808 patients recruited in the present study represents a relatively small sample size that may not be sufficient to detect significant differences between the subgroups (with RHR $>76 \mathrm{bpm}$ and RHR $<61 \mathrm{bpm}$, or with RHR 61$76 \mathrm{bpm}$ and $\mathrm{RHR}<61 \mathrm{bpm}$,) in terms of adverse outcomes. Secondly, the patients were not divided into more than three subgroups according to RHR because of the relatively small sample size. A larger population with more than three subgroups might provide more powerful evidence for identifying the cut-off points of RHR that related to clinical adverse outcome.

Our findings have several important clinical implications. The association between increasing RHR and MACEs strongly indicates that strict rate control may lead to the superior and beneficiary outcomes. Given the fact that the amount of patients is huge, additional adequately studies to identify optimal rate control strategies are warranted. Furthermore, there may be a floor effect or a threshold, below which lowering heart rate is no longer beneficial (and may be harmful). Thus, identifying the optimal threshold, and the optimal therapies to achieve that threshold, will require further investigation.

\section{Conclusions}

ACS patients after PCI with RHR $>76 \mathrm{bpm}$ were at higher risk of MACE during one-year follow-up compared with patients with RHR between 61 and $76 \mathrm{bpm}$. An elevated
RHR $\geq 61 \mathrm{bpm}$ was associated with significantly increased risk of one-year MACE in ACS patients.

Author contribution: S.W., K.C., and D.S. designed research; S.W., C.W., P.W., H.X., J.D. and D.Z. performed research; Z.G., L.Z., and C.F. contributed new reagents/ analytic tools. S.W., C.W., and D.S. analyzed data; and S.W., K.C., and D.S. wrote the paper.

\section{ACKNOWLEDGMENTS}

The authors would like to thank Hanjay for modification on earlier versions of this article. The authors are also grateful to Drs Feng Gu, Qiao-Ning Yang and Qiang Wang for their participation in clinical data collection and data loading. This work is supported by the "Eleventh Five-year Plan" for Sci \& Tech Research of China (No. 2006BA104A01).

\section{DECLARATION OF CONFLICTING INTERESTS}

The author(s) declared no potential conflicts of interest with respect to the research, authorship, and/or publication of this article.

\section{REFERENCES}

1. Cooney MT, Vartiainen E, Laatikainen T, Juolevi A, Dudina A, Graham IM. Elevated resting heart rate is an independent risk factor for cardiovascular disease in healthy men and women. Am Heart J 2010;159:612-19

2. Lang CC, Gupta S, Kalra P, Keavney B, Menown I, Morley C, Padmanabhan S. Elevated heart rate and cardiovascular outcomes in patients with coronary artery disease. Atherosclerosis 2010;212:1-8

3. Kolloch R, Legler UF, Champion A, Cooper-Dehoff RM, Handberg E, Zhou Q, Pepine CJ. Impact of resting heart rate on outcomes in hypertensive patients with coronary artery disease: findings from the International Verapamil-SR/trandolapril Study (INVEST). Eur Heart J 2008;29:1327-34

4. Khan H, Kunutsor S, Kalogeropoulos AP, Georgiopoulou VV, Newman AB, Harris TB, Bibbins-Domingo K, Kauhanen J, Gheorghiade M, Fonarow GC, Kritchevsky SB, Laukkanen JA, Butler J. Resting heart rate and risk of incident heart failure: three prospective cohort studies and a systematic meta-analysis. J Am Heart Assoc 2015;4:e001364

5. Saraiva F, Antonio N, Lourenço C, Gonçalves F, Monteiro P, Gonçalves L, Freitas M, Providência LA. Heart rate and prognosis in acute coronary syndromes. Rev Port Cardiol 2010;29:1101-19

6. Tekin K, Cagliyan CE, Tanboga IH, Balli M, Uysal OK, Ozkan B, Arik OZ, Cayli M. Influence of the timing of percutaneous coronary intervention on clinical outcomes in non-ST-elevation myocardial infarction. Korea Cir J 2013;43:725-30

7. Chinese Society of Cardiology of Chinese Medical Association, Editorial Board of Chinese Journal of Cardiology. Guideline for diagnosis and treatment of patients with unstable angina and non- ST-segment elevation myocardial infarction. Chin J Cardiol 2007;35:295-304

8. Antman EM, Hand M, Armstrong PW, Bates ER, Green LA, Halasyamani LK, Hochman JS, Krumholz HM, Lamas GA, Mullany CJ, Pearle DL, Sloan MA, Smith SC Jr, Anbe DT, Kushner FG, Ornato JP, Pearle DL, Sloan MA, Jacobs AK, Adams CD, Anderson JL, Buller CE, Creager MA, Ettinger SM, Halperin JL, Hunt SA, Lytle BW, Nishimura R, Page RL, Riegel B, Tarkington LG, Yancy CW. 2007 Focused update of the ACC/AHA 2004 guidelines for the management of patients with STelevation myocardial infarction. J Am Coll Cardiol 2008;51:210-47

9. World Medical Association declaration of Helsinki. Recommendations guiding physicians in biomedical research involving human subjects. JAMA 1997;277:925-6 
10. Legeai C, Jouven X, Tafflet M, Dartigues JF, Helmer C, Ritchie K, Amouyel P, Tzourio C, Ducimetière P, Empana JP. Resting heart rate, mortality and future coronary heart disease in the elderly: the 3C study. Eur J Cardiovasc Prev Rehabil 2010;18:488-97

11. Jensen MT, Marott JL, Jensen GB. Elevated resting heart rate is associated with greater risk of cardiovascular and all-cause mortality in current and former smokers. Int J Cardiol 2011;151:148-54

12. Paul L, Hastie CE, Li WS, Harrow C, Muir S, Connell JM, Dominiczak AF, McInnes GT, Padmanabhan S. Resting heart rate pattern during follow up and mortality in hypertensive patients. Hypertension 2010;55:567-74

13. Kovar D, Cannon CP, Bentley JH, Charlesworth A, Rogers WJ. Does initial and delayed heart rate predict mortality in patients with acute coronary syndromes. Clin Cardiol 2004;27:80-6

14. Timóteo AT, Toste A, Ramos R, Oliveira JA, Ferreira ML, Ferreira RC. Admission heart rate as a predictor of mortality in patients with acute coronary syndromes. Acute Cardiac Care 2011;13:205-10

15. Antoni ML, Boden H, Delgado V, Boersma E, Fox K, Schalij MJ, Bax JJ. Relationship between discharge heart rate and mortality in patients after acute myocardial infarction treated with primary percutaneous coronary intervention. Eur Heart J 2012;33:96-102

16. Disegni E, Goldbourt U, Reicher-Reiss H, Kaplinsky E, Zion M, Boyko $\mathrm{V}$, Behar S. The predictive value of admission heart rate on mortality in patients with acute myocardial infarction. SPRINT Study Group. Secondary Prevention Reinfarction Israeli Nifedipine Trial. J Clin Epidemiol 1995;48:1197-205

17. Zuanetti G, Mantini L, Hernández-Bernal F, Barlera S, di Gregorio D, Latini R, Maggioni AP. Relevance of heart rate as a prognostic factor in patients with acute myocardial infarction: insights from the GISSI-2 study. Eur Heart J 1998;19:F19-26

18. Parodi G, Bellandi B, Valenti R, Memisha G, Giuliani G, Velluzzi S, Migliorini A, Carrabba N, Antoniucci D. Heart rate as an independent prognostic risk factor in patients with acute myocardial infarction undergoing primary percutaneous coronary intervention. Atherosclerosis 2010;211:255-59
19. Fácila L, Morillas P, Quiles J, Soria F, Cordero A, Mazón P, Anguita M, Martín-Luengo C, Gonzalez-Juanatey JR, Bertomeu V, Investigators OB. Prognostic significance of heart rate in hospitalized patients presenting with myocardial infarction. World J Cardiol 2012;4:15-19

20. Okamura T, Hayakawa T, Kadowaki T, Kita Y, Okayama A, Elliott P, Ueshima H. Resting heart rate and cause-specific death in a 16.5-year cohort study of the Japanese general Population. Am Heart J 2004;147:1024-32

21. Kjekshus J. Importance of heart rate in determining beta-blocker efficacy in acute and long-term myocardial infarction intervention trials. Am J Cardiol 1986;57:43F-49F

22. Cucherat M. Quantitative relationship between resting heart rate reduction and magnitude of clinical benefits in post-myocardial infarction: a meta-regression of randomized clinical trials. Eur Heart J 2007;28:3012-19

23. Kelly A. Heart rate in coronary artery disease: should we lower it? Curr Treat Option Cardio Med 2013;15:118-28

24. Bangalore S, Messerli FH, Ou FS, Tamis-Holland J, Palazzo A, Roe MT, Hong MK, Peterson ED. CRUSADE Investigators: The association of admission heart rate and in-hospital cardiovascular events in patients with non-ST-segment elevation acute coronary syndromes: results from 135164 patients in the CRUSADE quality improvement initiative. Eur Heart J 2010;31:552-60

25. Åke H. Heart rate: an independent risk factor in cardiovascular disease. Eur Heart J 2006;27:2387-93

26. Traub O, Berk BC. Laminar shear stress - Mechanisms by which endothelial cells transduce an atheroprotective force. Arterioscl Throm Vas 1998;18:677-85

27. Sambuceti G, Marzilli M, Marraccini P, SchneiderEicke J, Gliozheni E, Parodi $\mathrm{O}$, LAbbate A. Coronary vasoconstriction during myocardial ischemia induced by rises in metabolic demand in patients with coronary artery disease. Circulation 1997;95:2652-59

(Received August 7, 2015, Accepted October 15, 2015) 